ARTÍCULO

\title{
Acciones del profesorado para una práctica inclusiva en la universidad
}

\author{
Noelia Melero Aguilar (1) \\ Anabel Moriña! (1) \\ Víctor-Hugo Perera' (1D
}

\section{RESUMEN}

En este artículo se analizan las acciones que el profesorado de una universidad española realiza para contribuir a la inclusión del alumnado con discapacidad. Para llevar a cabo el estudio realizamos entrevistas semiestructuradas a 20 docentes. Los resultados presentan diferentes acciones que el profesorado puso en práctica para ajustar la metodología, los materiales, y la evaluación. En las conclusiones, se plantea la necesidad de que en las asignaturas se contemplen actuaciones que hagan posible la respuesta a las necesidades derivadas de la discapacidad, así como se recomienda planificar los proyectos docentes desde los principios del diseño universal del aprendizaje.

PALABRAS CLAVE

educación inclusiva; enseñanza superior; profesorado.

'Universidad de Sevilla, Sevilha, España. 


\section{TEACHER ACTIONS FOR AN INCLUSIVE PRACTICE IN THE UNIVERSITY}

\section{ABSTRACT}

This paper analyzes the actions that the faculty of a Spanish university carries out to contribute to the inclusion of students with disabilities. To carry out the study, we conducted semi-structured interviews with 20 faculty members. Results present different actions that faculty members put into practice to adjust methodology, materials, and evaluation. The conclusion points out the need to subjects to contemplate actions that make possible the answer to the needs derived from the disability, as well as it recommends the planning of the syllabus from the principles of the universal design of learning.

KEYWORDS

inclusive education; higher education; faculty member.

\section{AÇÕES DO PROFESSOR PARA UMA PRÁTICA INCLUSIVA NA UNIVERSIDADE}

\section{RESUMO}

Este artigo tem como objetivo analisar as ações de professores de uma universidade espanhola em relação à inclusão de estudantes com deficiência. Para elaborar o estudo, foram feitas entrevistas semiestruturadas com 20 professores. Os resultados mostram que professores adotam diferentes ações, adaptadas à metodologia já existente, tais como materiais e avaliação. A conclusão mostra a necessidade permanente de contemplar ações que possibilitem a resposta às necessidades derivadas da deficiência, assim como se recomenda planejar projetos de ensino com base nos princípios do desenho universal da aprendizagem.

PALAVRAS-CHAVE

educação inclusiva; ensino superior; professorado. 


\section{INTRODUCCIÓN}

En los últimos años, las universidades están siendo protagonistas de un significativo avance en materia de inclusión educativa. En el caso de España, el acceso a la universidad de estudiantes con discapacidad es una realidad que está refrendada, tanto por la legislación nacional (por ejemplo, Ley Orgánica de Universidades 4/2007, Estatuto del Estudiante Universitario RD 1.791/2010 o Ley General de Derechos de las Personas con Discapacidad y de su Inclusión Social, 2013), como por diferentes estatutos y reglamentos regulados por iniciativa de las propias universidades.

En estas normativas se recogen los principios de igualdad de oportunidades, accesibilidad universal o diseño para todos. Sin embargo, en la práctica se ha estudiado que aún queda un largo camino por recorrer y existe un vacío legal en el que las acciones emprendidas dependen en muchas ocasiones de la buena voluntad de la comunidad universitaria (Castellana y Sala-Bars, 2006; Díaz y Funes, 2016; Muñoz-Cantero, Novo y Espiñeira, 2013). En este sentido, como Gibson (2015) o Thomas (2016) sugirieron, no es suficiente con garantizar el acceso a la universidad de estos estudiantes, teniendo en cuenta, como han concluido Sánchez-Gelabert y Elias (2017), que el abandono es más frecuente. Son necesarias políticas universitarias que accionen mecanismos para asegurar la permanencia y el éxito académico. En este sentido, en el ámbito de normativas específicas en materia de discapacidad, la universidad en la que se ha realizado este estudio, cuenta con un reglamento general de estudiantes (Universidad de Sevilla, 2009) donde se hace referencia a la creación de dos estructuras: la Unidad de Atención a Estudiantes con Discapacidad y la Comisión Técnica de Atención al Estudiante con Discapacidad. Por otro lado, existe una normativa específica en esta universidad sobre exención de importes por la prestación de servicios académicos y administrativos a los estudiantes con discapacidad (2008), y dos Planes integrales para personas con algún tipo de discapacidad (2009 y 2017).

En la mayoría de estudios, desde la voz del alumnado con discapacidad, se identifica al profesorado como una pieza clave para la inclusión de los estudiantes (Burgstahler, 2015; Mullins y Preyde,2013; Strnadová,Hájková y Květoňová, 2015). En términos de inclusión, en determinadas ocasiones, son necesarios revisar los proyectos docentes para facilitar el aprendizaje de todo el alumnado. En algunos estudios llevados a cabo se concluye que una de las principales barreras a las que tiene que enfrentarse este alumnado es la referida a los proyectos docentes no inclusivos y actitudes negativas por parte del profesorado (Garrison-Wade, 2012; Hewett et al., 2017; Hopkins, 2011; Leyser et al.,2011). Sakiz y Saricali (2017), por ejemplo, analizaron que las dificultades que experimentaban los estudiantes estaban relacionadas con planificaciones inflexibles y proyectos docentes homogéneos y estandarizados.

En algunas investigaciones se evidencia un gran desconocimiento por parte del profesorado, por un lado, hacia la discapacidad, término que según la Organización Mundial de la Salud (OMS, 2011) hace referencia a las deficiencias, las limitaciones de la actividad y las restricciones de la participación, a lo que autores como Gairín y Suárez (2014) añaden, además, la privación a las personas de su de- 
recho a participar plenamente en la sociedad y en la enseñanza superior. En España el concepto que de discapacidad se plantea es el recogido en la OMS (2011) y, por tanto, la discapacidad es comprendida como un fenómeno complejo que refleja una interacción entre las características del organismo humano (funciones sensoriales, intelectuales, motoras y emocionales de las personas) y las características de la sociedad en la que vive el individuo (como actitudes negativas, transporte y edificios inaccesibles, y falta de apoyo social). De modo que muchas de las limitaciones están relacionadas con el ambiente en el que viven las personas con discapacidad.

Por otro lado, las investigaciones evidencian que el profesorado no está formado en el uso de las metodologías pedagógicas más adecuadas para hacer frente a un aula diversa. En diferentes trabajos se plantea que el profesorado no está preparado para atender o dar respuesta a las necesidades de aprendizaje de estos estudiantes (Cardoso, 2013; Lovet et al., 2015; Moriña, Cortés y Molina, 2015; Moswela y Mukhopadhyay, 2011).

Respecto a la metodología utilizada por los docentes, autores como Fuller, Bradley y Healey (2004), resaltan los problemas que se encuentran algunos estudiantes con discapacidad durante el desarrollo de las clases. Entre los más comunes destacan las limitaciones a las que se enfrentan para tomar apuntes debido a la rapidez con la que los docentes explican, o la ausencia de participación e interacción en los procesos de enseñanza. Estudios como los de Holloway (2001) señalan cómo los estudiantes en diversas ocasiones demandan a los profesores que modifiquen sus estilos de enseñanza.

Otro elemento clave del currículo son las pruebas de evaluación que determinan la superación de las materias o asignaturas. Las investigaciones que han tratado esta cuestión señalan la dificultad que supone para el profesorado realizar modificaciones en los exámenes (Moswela y Mukhopadhyay, 2011). Pero, sobre todo, los estudios coinciden en resaltar que el profesorado se muestra reacio a plantear diversos modos de evaluación, priorizando la evaluación sumativa frente a la evaluación continua. Al profesorado le preocupa hacer estas modificaciones, ya que considera que podría estar dando un trato de favor (Burgstahler y Doe, 2006). Sin embargo, Hanafin et al. (2007) plantean el argumento contrario, llevar a cabo prácticas de evaluación más inclusivas, supone que los estudiantes con discapacidad estén en igualdad de condiciones.

Por otro lado, no siempre el profesorado es identificado como una barrera, ya que en otras ocasiones los estudios resaltan sus actitudes positivas hacia los estudiantes con discapacidad y reconocen su esfuerzo para dar respuesta a sus necesidades. Sin embargo, los docentes no siempre saben cómo apoyarlos (Venville, Street y Fossey, 2014). Una de las medidas que el profesorado puede llevar a cabo en su práctica docente para contribuir a la inclusión educativa es realizar los ajustes razonables que sean necesarios. Por ajuste razonable, de acuerdo con Fossey et al. (2017), se entiende una acción o modificación del currículo para ayudar a estos estudiantes a participar en los procesos de enseñanza y aprendizaje en igualdad de condiciones respecto al resto de sus compañeros.

No hay muchas investigaciones que se hayan centrado en estudiar los ajustes razonables que realiza el profesorado para incluir al alumnado con discapacidad 
(Fossey et al. 2017). Algunas, como el trabajo de Burgstahler y Doe (2006), concluyen que el profesorado se muestra dispuesto a realizar ajustes que no requieran un gran esfuerzo y que sean fáciles de llevar a cabo (por ejemplo, dar tiempo extra en los exámenes), pero presenta más resistencias para los ajustes que requieren una mayor implicación o dedicación de tiempo.

Schreuer y Sachs (2014) encontraron que cuando los estudiantes se beneficiaban de ajustes razonables repercutía positivamente en sus calificaciones. Esto lleva a pensar que ésta es una de las medidas que pueden ser tenidas en cuenta por las universidades para garantizar el éxito de los estudiantes. Por otro lado, otra de las acciones que deberían contemplarse es la planificación de proyectos docentes desde los principios del diseño universal de aprendizaje (DUA). De hecho, Marshak et al. (2010) consideran que los ajustes razonables podrían evitarse si los currículos se diseñaran desde los principios del diseño universal del aprendizaje. Si se adoptara éste, se deberían diseñar los productos y los entornos para ser utilizados por todos (Preiser y Smith, 2011; Rose y Meyer, 2006). Un proyecto docente diseñado universalmente está pensado desde el principio para tratar de satisfacer las necesidades educativas del mayor número de personas, haciendo innecesario el costoso proceso de introducir cambios en el currículo general, una vez diseñado, solo "para algunas personas".

ElDUA pretende eliminar la barrera de un currículo inflexible que dificulta la adquisición de aprendizajes por parte de estudiantes, pretendiendo que el currículo se ajuste a todo el alumnado. En este sentido, cuando se planifica un proyecto docente debe de existir una anticipación en el diseño de la asignatura, teniendo en cuenta que ésta será cursada por estudiantes con diferentes características y necesidades (Hitch, Dell y Larkin, 2016; Waitoller y Thorius, 2016).

De acuerdo con Mather y Muchatuta (2011), usar métodos de enseñanza y aprendizaje variados realza el compromiso del estudiante ya que cómo las personas aprenden puede diferir considerablemente. Beaudoin (2013) y Guasch y Hernández (2012) proponen que una combinación de clases magistrales con otras metodologías más activas (estudios de casos, debates o proyectos) puede involucrar más al alumnado en una asignatura.

Por otro lado, Guasch y Hernández (2012) recomiendan que se debería preparar materiales con un formato simple, intuitivo y coherente y proporcionarlos al alumnado con suficiente antelación para permitir un trabajo personal previo. Además, deberían estar disponibles tanto en formato impreso como en formato electrónico. Asimismo, sería conveniente preguntar específicamente al alumnado si tiene alguna dificultad para acceder a los materiales de la asignatura.

En cuanto a la evaluación, Martins, Morges y Gonçalves (2017) afirman que el profesorado realiza diferentes ajustes razonables relacionados con ésta. En el estudio de Mather y Muchatuta (2011) se plantean tres formas distintas de crear actividades de evaluación accesibles, a saber: evaluaciones alternativas (que implica proporcionar una evaluación completamente diferente para algunos estudiantes); arreglos adicionales (que conlleva la provisión de recursos adicionales, como por ejemplo el uso de un ordenador); y ajustes en la evaluación (que supone proporcionar variaciones a la implementación de la actividad de evaluación). 
Para finalizar, cabe resaltar que el corpus científico da muestra que el profesorado que basa su práctica docente en los principios de la educación inclusiva, beneficia no solo al alumnado con discapacidad, también al resto de estudiantes (Cunningham, 2013). Este artículo pretende cubrir algunas lagunas encontradas en la literatura científica, analizando cómo valoran la presencia de los estudiantes con discapacidad y cuáles son las acciones o ajustes razonables que el profesorado realiza cuando tiene alumnado con discapacidad en el aula.

\section{MÉTODO}

Los resultados que se presentan en este artículo forman parte de una investigación financiada por el Ministerio de Economía y Competitividad de España "eliminado título para una revisión anónima”. El propósito de este proyecto, con una duración de cuatro años (2014-2017) fue formar al profesorado universitario, a través de un curso basado en la modalidad de blended-learning (con una duración de 12 horas presenciales y 42 horas online), en cómo dar una respuesta educativa inclusiva al alumnado con discapacidad.

Uno de los temas que se analizaron en este proyecto de investigación fue la experiencia que los participantes en la formación tenían con estudiantes con discapacidad y las acciones o ajustes razonables que llevaron a cabo para incluirlos. Para ello, cuatro cuestiones de investigación guiaron el trabajo:

1. ¿Cómo valoraban las experiencias de dar respuesta a las necesidades de estudiantes con discapacidad?

2. ¿Cuáles eran las acciones o ajustes razonables que se realizan en relación a la metodología?

3. ¿Cuáles eran las acciones o ajustes razonables llevados a cabo en relación a los recursos?

4. ¿Qué acciones o ajustes razonables se hacían en relación a la evaluación?

En el estudio participaron 20 profesores de la misma universidad, y de diferentes áreas de conocimiento. A la muestra se accedió a través del centro de formación de la universidad, una vez que se dio publicidad al curso. Con el propósito de tener la mayor representatividad posible de profesorado, los criterios de selección fueron seis: docentes con o sin experiencia en alumnado con discapacidad en las aulas; profesorado de ambos géneros; diversidad con respecto a los años de experiencia de docencia del profesesorado; compromiso de introducir cambios en las aulas; disponibilidad para participar; representación de todos los campos de conocimiento (Ciencias de la Salud, Ciencias Experimentales, Enseñanzas Técnicas, Humanidades y Ciencias Sociales y Jurídicas). Sin embargo, este último criterio no se pudo cumplir al completo ya que en la formación no estuvo interesado en participar profesorado de dos ramas de conocimiento: Ciencias Experimentales y Enseñanzas Técnicas. Se ofertaron 30 plazas y en un principio 23 docentes se inscribieron en el curso. 
Finalmente, fueron 20 profesores los que participaron, ya que dos personas no comenzaron el curso y una tercera abandonó, una vez iniciada la formación.

En cuanto a las ramas de conocimiento a las que pertenecían los participantes, doce estaban vinculados al área de Ciencias Sociales y Jurídicas (de los cuales ocho eran docentes de la Facultad de Ciencias de la Educación), cuatro trabajaban en Ciencias de la Salud y otros cuatro en Humanidades. Doce eran mujeres y ocho eran hombres. Respecto a la experiencia previa con alumnado con discapacidad en el aula, 14 de los participantes declararon haber tenido al menos un alumno con discapacidad en su clase Entre la tipología se dieron casos de discapacidad visual y auditiva fundamentalmente, un caso de estudiante con parálisis cerebral y otro con síndrome de asperger. Por último, la mitad de los participantes contaban con cinco años o menos de experiencia en la universidad; y el resto con más años.

La metodología utilizada en este estudio fue cualitativa. Ya que como apuntan algunos autores, se rige por un diseño de investigación flexible, donde los estudios suelen comenzar con la formulación de algunos interrogantes, y donde el investigador observa el escenario y las personas desde una perspectiva holística (Flick, 2007; Lancy, 1993; Taylor y Bogdan, 1987). Creemos que esta opción metodológica es idónea para hacer oír las voces y narrar las experiencias de los participantes en el estudio.

Se recogió la información a través de entrevistas semi-estructuradas grupales e individuales. Algunas de las cuestiones de las entrevistas fueron: ¿Cuál es su experiencia con relación a estudiantes con discapacidad?, ¿puede señalar los ajustes que tuvo que realizar para responder a las necesidades de este alumnado?, ¿cómo hizo frente a las necesidades de este alumnado?

Para el análisis de datos, siguiendo la propuesta de Miles y Huberman (1994), se construyó un sistema de categorías y códigos que permitió organizar y comparar toda la información. Con el fin de facilitar el proceso de análisis se recurrió al programa informático MaxQDA12.

Por último, entre las cuestiones éticas de esta investigación se garantizó que la información fuera tratada de manera confidencial y anónima. Además, se les informó que en caso de decidir no continuar participando en el estudio, sus datos no se considerarían para el análisis y serían eliminados.

\section{RESULTADOS}

En líneas generales, los docentes se muestran satisfechos con sus experiencias en los procesos de enseñanza y aprendizaje con los estudiantes con discapacidad. Buena parte de las actuaciones llevadas a cabo estuvieron fuertemente marcados por una desmedida voluntad, un considerable esfuerzo y en ocasiones una gran dedicación por parte de los estudiantes y profesores que han participado de este tipo de experiencias. Para los docentes que desconocían todo lo relacionado con las medidas y actuaciones en materia de discapacidad, el grado de dificultad con el que se cursa una asignatura podía depender en gran medida de si el estudiante tenía claro el tipo de ayuda que necesitaba. 
En general, los docentes han aprendido de sus estudiantes que aún presentando alguna discapacidad, ésta no tiene porqué limitar sus múltiples competencias para seguir con normalidad el desarrollo de una clase. La mayoría de los estudiantes que no presentaron necesidades especiales en cuanto a la metodología que empleaba el docente y al material utilizado, sí requirieron de ciertos ajustes en las pruebas e instrumentos diseñados para evaluar sus aprendizajes. A excepción de este momento puntual en el proceso de aprendizaje de los estudiantes, la mayoría de los docentes consideraban que no tuvieron que realizar un trabajo extraordinario para adaptarse a los alumnos, que se implicaron bien y obtuvieron buenos resultados, similares a los de sus compañeros. A este respecto, los estudiantes han sido definidos con características positivas tales como que eran muy trabajadores, participativos y aplicados, así como que estaban muy motivados e interesados por su formación.

A continuación se describen aquellos ajustes que fueron realizados para favorecer la inclusión del alumnado con discapacidad. Éstos estaban referidos a la metodología docente, a los recursos de aprendizaje y a la evaluación.

\section{METODOLOGÍA DOCENTE PARA UNA PRÁCTICA EDUCATIVA INCLUSIVA EN EL AULA}

La práctica del docente en el aula estuvo metodológicamente condicionada por los distintos ajustes que se precisaron en respuesta a las necesidades educativas de aquellos estudiantes que presentaron algún tipo de discapacidad. En su actuación, los docentes adoptaron ciertas medidas relacionadas con el modo en cómo transmitir de manera efectiva su discurso en el aula. Algunas de las estrategias empleadas supusieron que el profesor limitara sus movimientos en la clase (como permanecer cerca del dispositivo de grabación situado sobre su mesa), situara su posición corporal en una dirección concreta (por ejemplo, manteniendo el contacto visual frente al alumno para que pudiese leer los labios), o modulara su ritmo y tono vocal (hablando más despacio y vocalizando mejor).

Él siempre se ponía en primera fila de la clase, y yo le daba mucho contacto visual e intentaba hablar más despacito. Es decir, vocalizaba un poquito mejor y, sobre todo, tenía mucho cuidado de no ponerme a escribir en la pizarra de espaldas, sino que, cuando estaba escribiendo, me callaba y luego me volvía y seguía con lo que fuese. A mí la verdad que me fue muy bien. (Profesor 13)

Otras medidas se aplicaron para facilitar el aprendizaje. De esta manera, los profesores emplearon estrategias metodológicas para explicar el significado de conceptos visuales e igualmente proporcionaron ejemplos cotidianos para hacer más comprensible los contenidos de cierta complejidad. Además, en ocasiones, fue preciso ajustar las actividades y dinámicas de trabajo en grupo tal que permitiesen una participación activa de estos estudiantes. En este punto, el grupo de compañeros 
de clase supieron incluir al estudiante con discapacidad asignándole roles que éstos podían desarrollar.

Uno de los espacios en los que la atención individualizada al alumnado tenía mayor presencia se encontraba en los despachos de los propios profesores. La actividad de tutorización desarrollada por los profesores suponía para estos estudiantes un valioso recurso para cubrir aquella información relevante de la materia de estudio que no se podía acceder en clase, recibir explicaciones sobre el desarrollo de las prácticas que no pudieron realizar o, simplemente, llevar a cabo un seguimiento sistematizado de los trabajos prácticos encomendados.

Entonces, en mi despacho yo le contaba la práctica, como son las prácticas... Le contaba lo que hacíamos, por ejemplo, en el temario de solubilidad, cuál era el objetivo, y cómo disolver azúcar en agua, cuánta cantidad tengo que echar... lo mismo que les cuento a todos mis alumnos en clase. (Profesor 17)

\section{APOYOS Y RECURSOS PARA EL APRENDIZAJE}

Una de las áreas en la que los profesores realizaron múltiples ajustes era los recursos para el aprendizaje. Gran parte de estas medidas pretendían facilitar al estudiante su acceso al currículo. Con este fin, se emplearon esencialmente recursos materiales y personales. En cuanto a los recursos materiales, los profesores estuvieron de acuerdo en que los estudiantes utilizaran dispositivos técnicos y tecnológicos como grabadoras de voz para registrar en clase las lecciones. Otros aparatos más avanzados permitieron obtener una transcripción directa en modo de apuntes. En un caso particular, se puso a disposición del estudiante casetes en donde el profesor grabó con su propia voz el contenido de libros de lectura obligatoria. Dado el tiempo que se requería para este tipo de ajustes, se delegó en la Organización Nacional de Ciegos Españoles (ONCE) la edición de muchos de estos materiales.

La persona que la acompañaba nos pidió permiso para grabar las clases. Ésta traía una especie de grabadora que situaba sobre su mesa. Además, con este dispositivo no sé si ella transcribía sus apuntes. Tenía también un altavoz. Yo le autoricé si problemas para que grabara la clase. (Profesor 16)

Los ajustes más comunes que realizó el profesorado sobre los materiales estaban relacionados con los contenidos audiovisuales y contenidos teóricos para el estudio. En este sentido, el profesor actuó buscando instrumentos adecuados a las necesidades educativas del estudiante; mientras que en otros casos recurrió a la ayuda externa para adaptar con recursos especializados, el material (por ejemplo,la adaptación de los textos a braille). Además, una medida recurrente adoptada por el profesorado era el envío por correo electrónico del material adaptado a título personal. Cuando el material no se había adaptado, el profesor lo solía enviar con antelación al correo electrónico del estudiante para que contara con tiempo suficiente para adaptarlo. 
Recibí una petición de una alumna para que le remitiera los materiales que íbamos a utilizar en clase, y yo se los envié con antelación a su correo electrónico para que la ONCE se los pudiera adaptar con tiempo. (Profesor 17)

En cuanto a los recursos personales, según relataban los participantes, los estudiantes recibieron ayudas del propio círculo familiar, de otros compañeros que tenían reconocido institucionalmente el papel de "alumno colaborador" (estudiante becado por la universidad que presta ayuda y acompañamiento a los estudiantes con discapacidad) o bien de técnicos especialistas de alguna institución (por ejemplo un intérprete de lenguaje de signos o técnico de la ONCE). Estas personas hacían de acompañantes y asistían a clases fundamentalmente para facilitar la toma de apuntes.

\section{MEDIDAS PARA LA EVALUACIÓN DE LOS APRENDIZAJES}

Por último, en el proceso de aprendizaje de los estudiantes, uno de los elementos del currículo que más atención recibía, y también mayor preocupación por parte del profesorado, era el de la evaluación. Un número notable de ajustes estaban referidos al acceso a la información, en este caso referida a la prueba de examen, que llevaron a cabo como medida preventiva y compensadora para facilitar al estudiante con discapacidad la adquisición del currículo. Concretamente, la mayoría de los profesores participantes en el estudio realizaron modificaciones en la evaluación. Éstas supusieron: adaptar el tiempo para el desarrollo de la prueba de examen, lo que normalmente implicaba no establecer un límite y ajustarse al tiempo que el estudiante necesitaba para finalizar la prueba; y adecuar el formato original de la prueba, que habitualmente era impreso, al formato digital, por ejemplo, permitiendo que para un estudiante con discapacidad visual pudiera ser accesible desde un ordenador, equipado con el software apropiado. En este tipo de ajustes, una medida alternativa consistía en realizar el examen oralmente y ajustar el texto, ampliando el tipo de letra para una mejor visibilidad de la grafía. Esta medida solía ir acompañada del empleo de instrumentos ópticos (por ejemplo una lupa) y elegir un lugar (por ejemplo el despacho del profesor) de fácil acceso, acondicionado para que el estudiante pudiera recibir una atención más personalizada de cara a la ejecución de la prueba de examen.

Escribe muy despacio. Entonces, le planteé las dos opciones: ¿cómo quieres hacerlo?, ¿escrito a mano o con el ordenador? Y me respondió que con el ordenador. Se trajo su portátil el día del examen y la situé junto a mi mesa. Y, además, le proyecté las imágenes el doble de tiempo. (Profesor 14)

Por otra parte, como medida excepcional, también en el momento de la evaluación se realizó puntualmente un ajuste en el que se priorizaron objetivos y suprimieron contenidos puntuales. Esta medida fue adoptada por un docente que entendió que el alumno no sería capaz de afrontar ciertos aprendizajes. 


\section{CONCLUSIONES Y DISCUSIÓN}

El estudio llevado a cabo analiza las experiencias de docentes universitarios para incluir a alumnado con discapacidad y las acciones llevadas a cabo. Lourens y Swartz (2016) o Naik (2017) estudiaron, desde la perspectiva del alumnado, ciertas acciones que se pueden realizar en el aula. Sin embargo, este artículo presenta los ajustes desde la perspectiva del profesorado. Esta es la principal aportación del estudio, ya que no es frecuente encontrar trabajos científicos sobre el análisis de estos ajustes desde la perspectiva del profesorado. Más en concreto, en España, no contamos con suficientes trabajos centrados en analizar la figura del profesor universitario en relación a su experiencia con alumnado con discapacidad y las acciones emprendidas para atender a las necesidades de estos estudiantes. De las investigaciones realizadas, cabe destacar la de Castellana y Sala-Bars (2006) en la que participaron profesores de diferentes áreas de conocimiento. En este trabajo se concluye que la presencia de estos estudiantes genera inseguridad e incomodidad. Muchos profesores realizan adaptaciones curriculares cuando son necesarias, pero la mayoría de los docentes sienten que no están informados ni formados en discapacidad. Respecto a esta última idea Sánchez Palomino (2011) llegó a una conclusión similar. En otro trabajo, Martínez y Bilbao (2011) analizaron las actitudes de los docentes hacia los estudiantes con discapacidad.

Respecto a nuestro estudio, en primer lugar, cabe destacar que los participantes, a pesar de tener que replantear la organización y diseño de su asignatura, o tener que realizar algún tipo de modificación en alguno de estos aspectos mencionados, mostró una relación muy positiva con el alumnado con discapacidad y una buena voluntad para realizar los ajustes necesarios en su práctica docente (Becker y Palladino, 2016; Yssel, Pak y Neilke, 2016).

La valoración de estos docentes con respecto a este alumnado ha sido también muy positiva, estimando su compromiso, esfuerzo e implicación, y los buenos resultados académicos que obtuvieron. Reconociendo, además, ser conscientes del papel y apoyo tan determinante que ejercen con respecto a estos estudiantes. Como argumentan Lombardi y Murray (2011), el profesorado juega un papel fundamental en el desarrollo académico de este alumnado, por lo que sus actitudes y sus prácticas son determinantes en su éxito o fracaso.

En relación a los ajustes razonables, en lo que se refiere a la metodología utilizada en el aula, los resultados del estudio determinan la preocupación del profesorado por transmitir de manera efectiva su discurso en el aula. El docente, al igual que se concluyó en el trabajo de Fuller, Bradley y Healey (2004), es consciente de que su metodología no llega a todo el alumnado y se esfuerza por emplear diversas estrategias metodológicas para explicar y hacer más comprensible los contenidos de cierta complejidad. En esta línea, Marcelo (2013) reconoce la importancia del profesorado como agende innovador, con capacidad para introducir cambios en sus prácticas habituales, en beneficio de las posibilidades del aprendizaje de los estudiantes. 
Por otra parte, realiza importantes ajustes en cuanto a las actividades y dinámicas de trabajo en grupo que permita una participación más activa de estos estudiantes. En este sentido, el grupo de compañeros de clase, también ejerce un papel importante, mostrando un significativo apoyo, al organizar el trabajo en grupo asignando roles y tareas asequibles y acordes a las características de este alumnado. En los trabajos de Castellana y Sala-Bars (2006) y Moswela y Mukhopadhyay (2011), los compañeros también son identificados como imprescindibles para el aprendizaje de este alumnado.

En relación a la acción tutorial, puede considerarse como un valioso recurso. Ésta puede ser concebida como un espacio donde poder cubrir y completar aquellas informaciones relevantes de la materia que no han podido registrarse en el aula. Por lo tanto, al igual que reconocieran Gairín et al. (2013), que las universidades cuenten con planes de acción tutorial puede facilitar la inclusión del alumnado con discapacidad.

Con relación a los ajustes razonables en los recursos utilizados por el profesorado en su práctica docente, en el estudio se ha realizado una distinción entre recursos materiales y personales. En cuanto a los recursos materiales, uno de los logros, muy valorado por el alumnado, ha sido el que los docentes estuvieran de acuerdo en ser grabados durante las sesiones de clase. El alumnado pudo utilizar dispositivos técnicos y tecnológicos como grabadoras de voz para registrar en clase las lecciones impartidas, facilitando que el alumnado pueda contar con todas las explicaciones y ejemplos literales expuestos en clase, lo que facilita significativamente para este alumnado llevar el hilo secuencial de las sesiones de clase, y tener una visión global de la asignatura (Lombardi y Murray, 2011; Wolman et al. 2004). Esta conclusión resulta significativa, ya que en otros estudios, los estudiantes denuncian precisamente la negativa del profesorado a que se utilice en sus clases apoyos adicionales (Hewett et al., 2017).

En resumen, realizar ajustes en los recursos y dispositivos audiovisuales que los docentes utilizan en el desarrollo de sus clases es realmente positivo para el desarrollo académico de estos estudiantes (Guasch y Hernández, 2012; Lersilp, 2016). Coincidimos con Naik (2017) en que realizar ajustes razonables en estos instrumentos, supone una gran ayuda para este alumnado.

En cuanto a los recursos personales, los docentes valoran y apoyan las ayudas recibidas por el círculo familiar, así como de otras figuras como; el alumno colaborador o bien, el intérprete de lengua de signos. Personas que han ejercido de acompañantes al aula, a las tutorías, y que asistían a clase fundamentalmente para facilitar la toma de apuntes. Acciones muy útiles que han supuesto resultados muy positivos para estos estudiantes, como también apareció en los estudios de Lombardi, Murray y Kowitt (2016), Lourens y Swartz (2016) o Phillips et al. (2012).

Finalmente, en lo que se refiere al seguimiento y evaluación de los aprendizajes, los docentes reparan en la importancia que el alumnado de manera general suele otorgar a la evaluación de sus asignaturas, a la manera en que se les va a evaluar, y al contenido que se va a evaluar, provocando dudas, conjeturas y diversos planteamientos cada vez que se abordan estas cuestiones en clase. Esto les lleva a reflexionar 
y tomar conciencia de los interrogantes e inseguridades que puede despertar la evaluación en el alumnado con discapacidad, teniendo en cuenta las dificultades que estos estudiantes puedan tener. Por ello, los ajustes en la evaluación es de las medidas más comunes y variadas llevadas a cabo por el profesorado, coincidiendo con otros investigadores que defienden la importancia de utilizar una variedad de tareas de evaluación (Mather y Muchatuta, 2011). Resulta significativo la flexibilidad del profesorado en cuanto a las necesidades del alumnado, garantizando que las pruebas se realicen en igualdad de condiciones con el resto de los estudiantes, como así lo refrendan otros estudios (Baker, Boland y Nowik, 2012; Hanafin et al., 2007; Lombardi y Murray, 2011).

En cuanto a los avances epistemológicos que supone la investigación para la inclusión en la enseñanza superior española, por un lado, el estudio se lleva a cabo con docentes universitarios de diferentes áreas de conocimiento de una universidad española, resaltando aquí el carácter interdisciplinar del mismo. Por otra parte, aporta resultados desde la visión de docentes del ámbito de la educación superior, y no desde la visión de los estudiantes, como ha sido habitual en el contexto español (Castellana y Sala-Bars, 2006; Moriña y Perera, 2018). Además, la investigación aporta acciones concretas y buenas prácticas que los docentes, contemplan en sus proyectos docentes, lo que lo convierte en un referente, aportando evidencias para seguir construyendo una universidad inclusiva, tanto en el contexto español como en otros contextos internacionales.

La realización de ajustes en el currículo de la educación superior es un garante de éxito en las trayectorias universitarias de los estudiantes con discapacidad. No obstante, se requiere un paso hacia delante y que los docentes no solo realicen ajustes, y planifiquen sus proyectos docentes desde los principios del DUA. Para ello sería recomendable que las universidades fomentaran acciones para apoyarlos y formar al profesorado en éste (Nancy et al., 2017). Pensar los proyectos desde el DUA evitaría la inversión de tiempo que se dedica a realizar los ajustes, una vez iniciadas las asignaturas, actuando proactiva, antes que reactivamente. Por último, conviene recordar que basar la práctica docente en estos principios, beneficiaría no sólo al alumnado con discapacidad, también al resto de estudiantes, contribuyendo así a construir un escenario universitario basado en la inclusión y el respecto a la diversidad (Cunningham, 2013).

\section{REFERENCIAS}

BAKER, K. Q.; BOLAND, K.; NOWIK, C. M. A campus survey of faculty and student perceptions of persons with disabilities. Journal of Postsecondary Education and Disability, Huntersville, NC, v. 25, n. 4, p. 309-329, 2012.

BEAUDOIN, J. P. Introduction to inclusive teaching practices. Ottawa: University of Ottawa, 2013. 
BECKER, S.; PALLADINO, J. Assessing faculty perspectives about teaching and working with students with disabilities. Journal of Postsecundary Education and Disability, Huntersville, NC, v. 29, p. 65-82, 2016.

BURGSTAHLER, S. Opening doors or slamming them shut? Online learning practices and students with disabilities. Social Inclusion, Lisboa, v. 3, n. 6, p. 69-79, 2015. http://dx.doi.org/10.17645/si.v3i6.420

BURGSTAHLER, S.; DOE, T. Improving postsecondary outcomes for students with disabilities: designing professional development for faculty. Journal of Postsecondary Education and Disability, Huntersville, NC,v. 18, n. 2, p. 135-147, 2006. Disponible en: http://www.ahead-archive.org/uploads/docs/jped/journals/JPEDVo118No2.rtf. Acceso en: 29 abr. 2019.

CARDOSO, R. M. Política de educação especial na perspectiva inclusiva e a formação docente no Brasil. Revista Brasileira de Educação, Rio de Janeiro, v. 18, n. 52, p. 101119, 2013. http://dx.doi.org/10.1590/S1413-24782013000100007

CASTELLANA, M.; SALA-BARS, I. Estudiantes con discapacidad en la universidad. Barcelona: Fundació Blanquerna Assistencial i de Serveis, 2006.

CUNNINGHAM, S. Teaching a diverse student body - a proposed tool for lecturers to self-evaluate their approach to inclusive teaching. Practice and Evidence of the Scholarship of Teaching and Learning in Higher Education, Bristol, v. 8, n. 1, p. 3-27, 2013.

DÍAZ, V.; FUNES, S. Universidad inclusiva: reflexiones a partir de las experiencias de estudiantes con discapacidad de una universidad pública madrileña. Prisma Social, Madrid, v. 16, p. 450-494, 2016.

ESPAÑA. Ley Orgánica de Universidades 4/2007, de 12 de abril, por la que se modifica la Ley Orgánica 6/2001, de 21 de diciembre. Boletín Oficial del Estado, Madrid, n. 89, 13 abr. 2007.p. 16.241-16.260. Disponible en: https:/www.boe.es/boe/ dias/2007/04/13/pdfs/A16241-16260.pdf. Acceso en: 20 feb. 2019.

ESPAÑA. Real decreto 1.791/2010, de 30 de diciembre, por el que se aprueba el Estatuto del Estudiante Universitario. Boletín Oficial del Estado, Madrid, n. 318, 31 dic.2010. p. 10.9353-10.9380. Disponible en: https://www.boe.es/boe/dias/2010/12/31/ pdfs/BOE-A-2010-20147.pdf. Acceso en: 20 feb. 2019.

ESPAÑA. Real decreto legislativo 1/2013, de 29 de noviembre, por el que se aprueba el Texto Refundido de la Ley General de Derechos de las Personas con Discapacidad y de su Inclusión Social. Boletín Oficial del Estado, Madrid, n. 289, 3 dic. 2013. p. 95.635-95.637. Disponible en: https://www.boe.es/boe/dias/2013/12/03/pdfs/ BOE-A-2013-12632.pdf. Acceso en: 20 feb 2019.

FLICK, U. Introducción a la investigación cualitativa. Madrid: Morata, 2007. FOSSEY, E.; CHAFFEY, L.; VENVILLE, A.; ENNALS, P.; DOUGLASD,J.; BIGBY, C. Navigating the complexity of disability support in tertiary education: perspectives of students and disability service staff. International Journal of Inclusive Education, United Kingdom, v. 21, n. 8, 2017. https://doi.org/10.1080/13603116.2017.1278798 
FULLER, M.; BRADLEY, A.; HEALEY, M. Incorporating disabled students within an inclusive higher education environment. Disability \& Society, United Kingdom, v. 19, n. 5, p. 455-468, 2004. https://doi.org/10.1080/0968759042000235307

GARRISON-WADE, D. F. Listening to their voices: factors that inhibit or enhance postsecondary outcomes for students' with disabilities. International Journal of Special Education, Toronto, v. 27, n. 2, p. 113-125, 2012.

GAIRÍN, J.; SUÁREZ, C. I. Clarificar e identificar a los grupos vulnerables. In: GAIRÍN, J. (coord.). Colectivos vulnerables en la universidad. Reflexiones y propuestas para la intervención. Madrid: Wolters Kluwer España, 2014. p. 35-61.

GAIRÍN, J.; MUÑOZ, J. L.; GALAN, A.; SANAHUJA, J. M.; FERNÁNDEZ, M. Planes de acción tutorial para estudiantes con discapacidad: una propuesta para mejorar la calidad formativa en las universidades españolas. Revista Iberoamericana de Educación, Madrid, v. 63, p. 115-126, 2013.

GIBSON, S. When rights are not enough: what is? Moving towards new pedagogy for inclusive education within UK universities. International Journal of Inclusive Education, United Kingdom, v. 19, n. 8,p. 875-886, 2015. https://doi.org/10.1080/13603116.2015.1015177

GUASCH, D.; HERNÁNDEZ, J. (coord.). Guía para implementar el Universal Instructional Design - UID (diseño instruccional universal) en la universidad. Barcelona: Observatorio Universidad y Discapacidad, 2012.

HANAFIN, J.; SHEVLIN, M.; KENNY, M.; MC NEELA, E. Including young people with disabilities: Assessment challenges in higher education. Higher Education, Netherlands, v. 54, p. 435-448, 2007. https://doi.org/10.1007/s10734-006-9005-9

HEWETT, R.; DOUGLAS, G.; MCLINDEN, M.; KEIL, S. Developing an inclusive learning environment for students with visual impairment in higher education: progressive mutual accommodation and learner experiences in the United Kingdom. European Journal of Special Needs Education, United Kingdom, v. 32, n. 1, p. 89-109, 2017. https://doi.org/10.1080/08856257.2016.1254971

HITCH, D.; DELL, K.; LARKIN, H. Does universal design education impact on the attitudes of architecture students towards people with disability? Journal of Accessibility and Design for All, Barcelona, v. 6, n. 1, p. 26-48, 2016. https://doi.org/10.17411/jacces.v6i1.103

HOPKINS, L. The path of least resistance: a voice-relational analysis of disabled students' experiences of discrimination in English universities. International Journal of Inclusive Education, United Kingdom, v. 15, p. 711-727, 2011. https://doi.org/10.1080/13603110903317684

HOLLOWAY, S. The experience of higher education from the perspective of disabled students. Disability \& Society, United Kingdom, v. 16, p. 597-615, 2001. https://doi.org/10.1080/09687590120059568

LANCY, D. F. Qualitative research in education. An introduction to the major traditions. New York: Longman, 1993.

LERSILP, T. Assistive technology and educational services for undergraduate students with disabilities at universities in the Northern Thailand. 
Procedia Environmental Sciences, Netherlands, v. 36, p. 61-64, 2016. https://doi.org/10.1016/j.proenv.2016.09.012

LEYSER, Y.; GREENBERGER, L.; SHARONI, V.; VOGEL, G. Students with disabilities in teacher education: changes in faculty attitudes toward accommodations over ten years. International Journal of Special Education, Canada, v. 26, n. 1, p. 162-174, 2011.

LOMBARDI, A. R.; MURRAY, C. Measuring university faculty attitudes toward disability: willingness to accommodate and adopt universal design principles. Journal of Vocational Rehabilitation, Netherlands, v. 34, p. 43-56, 2011. https://doi.org/10.3233/JVR-2010-0533

LOMBARDI,A.; MURRAY, C.; KOWITT,J. Social support and academic success for college students with disabilities: do relationship types matter? Journal of Vocational Rehabilitation, Netherlands, v. 44, p. 1-13,2016. https://doi.org/10.3233/JVR-150776 LOURENS, H.; SWARTZ, L. Experiences of visually impaired students in higher education: bodily perspectives on inclusive education. Disability \& Society, United Kingdom, v. 31, n. 2, p. 240-251, 2016.

LOVET, T. S.; KRESIER, N.; CAMARGO, E.; GRUBBS, M.; KIN, E. J.; BURGE, P. L.; CULVER, S. M. STEM faculty experiences with students with disabilities at a land grant institution. Journal of Education and Training Studies, Netherlands, v. 3, n. 1, p. 27-38, 2015. https://doi.org/10.11114/jets.v3i1.573

MARCELO, C. Las tecnologías para la innovación y la práctica docente. Revista Brasileira de Educação, Rio de Janeiro, v. 18, n. 52, p. 25-47, 2013. http://dx.doi.org/10.1590/S1413-24782013000100003

MARSHAK, L.; VAN WIEREN,T.; RAEKE-FERRELL,D.; SWISS, L.; DUGAN, C. Exploring barriers to college student use of disability services and accommodations. Journal of Postsecondary Education and Disability, Huntersville, NC, v. 22, n. 3, p. 151-165, 2010. MARTÍNEZ, M. A.; BILBAO, M. C. Los docentes de la Universidad de Burgos y su actitud hacia las personas con discapacidad. Revista Española de Discapacidad Intelectual, Madrid, v. 42, n. 4, p. 50-78, 2011.

MARTINS, M. E.; MORGES, M. L.; GONÇALVES, T. Attitudes towards inclusion in higher education in a Portuguese University. International Journal of Inclusive Education, United Kingdom, p. 1-16, 2017. https://doi.org/10.1080/13603116.2017.1377299

MATHER, G.; MUCHATUTA, M. How to teach with inclusive practice. Learning through diversity. Sydney: Macquarie University, 2011.

MILES, M. B.; HUBERMAN, A.M. Qualitative data analysis. 2. ed. Thousand Oaks, CA: Sage Publications, 1994.

MORIÑA,A.; CORTÉS,M.D.; MOLINA,V.What if we could imagine the ideal professor? Proposals for improvement by university students with disabilities. Teaching and Teacher Education, Amsterdam, n. 52, p. 91-98, 2015. https://doi.org/10.1016/j.tate.2015.09.008

MORIÑA, A.; PERERA, V. H. Inclusive higher education in Spain: students with disabilities speak out. Journal of Hispanic Higher Education, Thousand Oaks, 28 May 2018. https://doi.org/10.1177/1538192718777360 
MOSWELA, E.; MUKHOPADHYAY, S. Asking for too much? The voices of students with disabilities in Botswana. Disability \& Society, United Kingdom, v. 26, p. 307-319, 2011. https://doi.org/10.1080/09687599.2011.560414

MULLINS, L.; PREYDE, M. The lived experience of students with an invisible disability at a Canadian university. Disability \& Society, United Kingdom, v. 28, n. 2, p. 147-160, 2013. https://doi.org/10.1080/09687599.2012.752127

MUÑOZ-CANTERO,J.; NOVO, I.; ESPIÑEIRA, E. La inclusión de los estudiantes universitarios presenciales: actitudes e intención de apoyo por parte de sus compañeros. Estudios sobre Educación, Pamplona, v. 24, p. 103-124, 2013.

NAIK, N. Dual PowerPoint presentation approach for students with special educational needs and note-takers. European Journal of Special Needs Education, United Kingdom, v. 32, n. 1, p. 146-152, 2017. https://doi.org/10.1080/08856257.2016.1254970

NANCY, J.; ELLEN, M.; KIRSTEN, R.; AUTUMN, K. Disability in higher education. A Social Justice Approach. San Francisco, CA: Jossey-Bass, 2017.

ORGANIZACIÓN MUNDIAL DE LA SALUD. Informe mundial sobre la discapacidad. Ginebra: OMS, 2011. Disponible en: https://www.who.int/disabilities/ world_report/2011/summary_es.pdf. Acceso en: 20 feb. 2019.

PHILLIPS, A.; TERRAS, K.; SWINNEY, L.; SCHNEWEIS, C. Online disability accommodations: faculty experiences at one public university. Journal of Postsecondary Education and Disability, Huntersville, NC, v. 25, n. 4, p. 331-344, 2012.

PREISER, W. F. E.; SMITH, K. H. (ed.). Universal design handbook. New York: McGraw-Hill, 2011.

ROSE, D. H.; MEYER, A. (ed.). A practical reader in universal design for learning. Cambridge, MA: Harvard Education Press, 2006.

SAKIZ, H.; SARICALI, M. Including students with visual difficulty within higher education: necessary steps. Exceptionality, United States, p. 266-282, 2017. https:// doi.org/10.1080/09362835.2017.1283627

SÁNCHEZ-GELABERT, A.; ELIAS, M. Los estudiantes universitarios no tradicionales y el abandono de los estudios. Estudios sobre Educación, Pamplona, v. 32, 27-48, 2017. https://doi.org/10.15581/004.32.27-48

SÁNCHEZ PALOMINO, A.La Universidad de Almería ante la integración educativa y social de los estudiantes con discapacidad: Ideas y actitudes del personal docente e investigador. Revista de Educación, Madrid, n. 354, p. 575-603, enero/abr. 2011.

SCHREUER, N.; SACHS, D. Efficacy of accommodations for students with disabilities in higher education. Journal of Vocational Rehabilitation, Netherlands, v. 40, n. 1, p. 27-40, 2014. https://doi.org/10.3233/JVR-130665

STRNADOVÁ, I.; HÁJKOVÁ, V.; KVĚTOŇOVÁ, L. Voices of university students with disabilities: inclusive education on the tertiary level — a reality or a distant dream? International Journal of Inclusive Education, United Kingdom, v. 19, n. 10, p. 1.0801.095, 2015. https://doi.org/10.1080/13603116.2015.1037868 
TAYLOR, S. J.; BOGDAN, R. (ed.). Introducción a los métodos cualitativos de investigación. Barcelona: Paidós, 1987.

THOMAS, L. Developing inclusive learning to improve the engagement, belonging, retention, and success of students from diverse groups. In: SHAH, M.; BENNETT, A.; SOUTHGATE, E. (ed.). Widening higher education participation. A global perspective. Oxford: Elsevier, 2016. p. 135-159.

UNIVERSIDAD DE SEVILLA. Unidad de Atención a Estudiantes con Discapacidad. s/f. Disponible en: https://sacu.us.es/sacu-quehacemos-uaed. Acceso en: 19 feb. 2019.

UNIVERSIDAD DE SEVILLA. Resolución de 17 de junio de 2008 sobre exención de importes a satisfacer por la prestación de servicios académicos y administrativos a los estudiantes discapacitados. Boletín Oficial de la Universidad de Sevilla, n. 4, 5 nov. 2008. p. 20-22. Disponible en: http://bous.us.es/2008/numero-4/pdf/archivo-8.pdf. Acceso en: 20 feb. 2019.

UNIVERSIDAD DE SEVILLA. Acuerdo 10.1/CG 29-9-09 por el que se aprueba el I Plan Integral para Personas con algún tipo de discapacidad. Boletín Oficial de la Universidad de Sevilla, n. 9, 16 oct. 2009. p. 57-59. Disponible en: http://bous.us.es/2009/numero-9/pdf/archivo-7.pdf. Acceso en: 20 feb. 2019.

UNIVERSIDAD DE SEVILLA. Reglamento General de Estudiantes de la Universidad de Sevilla, Universidad de Sevilla. Boletín Oficial de la Universidad de Sevilla, n. 4, 1 abr. 2009. p. 24-35. Disponible en: http://bous.us.es/2009/numero-4/pdf/archivo-3.pdf. Acceso en: 20 feb. 2019.

UNIVERSIDAD DE SEVILLA. Acuerdo 9.1/CG 23-6-17, por el que se aprueba el II Plan Integral de personas con algún tipo de discapacidad. Boletín Oficial de la Universidad de Sevilla, n. 4, 24 jul. 2017. p. 732-738. Disponible en: http://bous.us.es/2017/BOUS-04-2017/pdf/BOUS04.pdf. Acceso en: 20 feb. 2019.

VENVILLE, A.; STREET, A.; FOSSEY,E. Student perspectives on disclosure of mental illness in post-compulsory education: displacing doxa. Disability \& Society, United Kingdom, v. 29, n. 5, p. 792-806, 2014. https://doi.org/10.1080/09687599.2013.844101 WAITOLLER, F. R.; THORIUS, K. A. K. Cross-pollinating culturally sustaining pedagogy and universal design for learning: toward an inclusive pedagogy that accounts for dis/ability. Harvard Educational Review, Cambridge, MA, v. 86, n. 3, p. 366-389, 2016. https://doi.org/10.17763/1943-5045-86.3.366

WOLMAN, C.; SUAREZ, C.; FIGUEROA, S.; HARRIS-LOOBY, J. The accommodation of university students with disabilities inventory (AUSDI): assessing American and Mexican faculty attitudes toward students with disabilities. Journal of Hispanic Higher Education, v. 3, n. 3, p. 284-295, 2004. https://doi.org/10.1177/1538192704265985

YSSEL, N.; PAK, N.; BEILKE, J. A door must be opened: perceptions of students with disabilities in higher education. International Journal of Disability, Development and Education, United Kingdom, p. 1-11, 2016. https://doi.org/10.1080/1034912X.2015.1123232 


\section{SOBRE LOS AUTORES}

Noelia Melero Aguilar es doctora en pedagogía por la Universidad de Sevilla (España). Profesora de la misma institución.

E-mail: nmelero@us.es

Anabel Moriña es doctora en pedagogía por la Universidad de Sevilla (España). Profesora de la misma institución.

E-mail:anabelm@us.es

Víctor Hugo Perera es doctor en pedagogía (didáctica y organización educativa) por la Universidad de Sevilla (España). Profesor de la misma institución. E-mail:vhperera@us.es

Recibido el 04 de agosto de 2018 Aprobado el 13 de diciembre de 2018 